OPEN ACCESS

Edited by:

Steve Lindemann,

Pacific Northwest National

Laboratory, USA

Reviewed by:

Antonis Chatzinotas,

Helmholtz Centre for Environmental

Research - UFZ, Germany

Amélia M. Sarmento,

Universidade Fernando Pessoa,

Portugal

*Correspondence:

Ehsan Khafipour

ehsan.khafipour@umanitoba.ca;

Natalia Elguezabal

nelguezabal@neiker.eus

Specialty section:

This article was submitted to

Systems Microbiology,

a section of the journal

Frontiers in Microbiology

Received: 30 November 2015 Accepted: 18 March 2016

Published: 31 March 2016

Citation

Arrazuria R, Elguezabal N, Juste RA, Derakhshani H and Khafipour E (2016)

Mycobacterium avium Subspecies paratuberculosis Infection Modifies Gut Microbiota under Different Dietary

Conditions in a Rabbit Model.

Front. Microbiol. 7:446.

doi: 10.3389/fmicb.2016.00446

\section{Mycobacterium avium Subspecies paratuberculosis Infection Modifies Gut Microbiota under Different Dietary Conditions in a Rabbit Model}

\author{
Rakel Arrazuria', Natalia Elguezabal1*, Ramon A. Juste', Hooman Derakhshani² and \\ Ehsan Khafipour ${ }^{2,3 *}$
}

${ }^{1}$ Department of Animal Health, NEIKER-Instituto Vasco de Investigación y Desarrollo Agrario, Derio, Spain, ${ }^{2}$ Department of Animal Science, University of Manitoba, Winnipeg, MB, Canada, ${ }^{3}$ Department of Medical Microbiology, University of Manitoba, Winnipeg, MB, Canada

Mycobacterium avium subspecies paratuberculosis (MAP) the causative agent of paratuberculosis, produces a chronic granulomatous inflammation of the gastrointestinal tract of ruminants. It has been recently suggested that MAP infection may be associated with dysbiosis of intestinal microbiota in ruminants. Since diet is one of the key factors affecting the balance of microbial populations in the digestive tract, we intended to evaluate the effect of MAP infection in a rabbit model fed a regular or high fiber diet during challenge. The composition of microbiota of the cecal content and the sacculus rotundus was studied in 20 New Zealand white female rabbits. The extracted DNA was subjected to paired-end Illumina sequencing of the V3-V4 hypervariable region of the 16S rRNA gene for microbiota analysis. Microbial richness (Chao1) in the cecal content was significantly increased by MAP infection in regular diet rabbits $(p=0.0043)$ and marginally increased $(p=0.0503)$ in the high fiber group. Analysis of beta-diversity showed that MAP infection produces deeper changes in the microbiota of sacculus rotundus than in the cecal content. A lower abundance of Proteobacteria in the cecal content of infected animals fed the high fiber diet and also lower abundance of Bacteroidetes in the sacculus rotundus of infected animals fed the regular diet were observed. Based on OPLS-DA analysis, we observed that some bacteria repeatedly appear to be positively associated with infection in different samples under different diets (families Dehalobacteriaceae, Coriobacteriaceae, and Mogibacteriaceae; genus Anaerofustis). The same phenomenon was observed with some of the bacteria negatively associated with MAP infection (genera Anaerostipes and Coprobacillus). However, other groups of bacteria (Enterobacteriaceae family and ML615J-28 order) were positively associated with infection in some circumstances and negatively associated with infection in others. Data demonstrate that MAP infection and diet changes do interact and result in shifts in the microbiota of the cecal content and sacculus rotundus of rabbits.

Keywords: Mycobacterium avium subsp. paratuberculosis, rabbit, animal model, high fiber diet, gut microbiota, inflammation 


\section{INTRODUCTION}

Mycobacterium avium subspecies paratuberculosis (MAP) is the causative agent of paratuberculosis (PTB) a chronic granulomatous inflammation of the gastrointestinal (GI) tract of ruminants. The infection may be cleared by the host or develop into subclinical or clinical disease causing significant economic losses in livestock (Clarke, 1997; Kennedy and Benedictus, 2001).

Although PTB has been historically linked to domestic ruminants, wild-life ruminant, and non-ruminant species are also susceptible to infection (Chiodini and Van Kruiningen, 1983; Beard et al., 2001a; Forde et al., 2013). In addition, association of MAP with Crohn's disease (CD; Naser et al., 2004; Juste et al., 2008) and Type 1 diabetes (Masala et al., 2011; Naser et al., 2013) in humans adds further interest to the role of this disease as a potential zoonosis.

It is known that the gut microbiome plays an important role in competitive exclusion of pathogens and in development and maturation of intestinal mucosal immunity (Kau et al., 2011; Stecher and Hardt, 2011). Many studies have documented differences in the composition of host associated microbial communities between healthy and diseased states (Clemente et al., 2012; Karlsson et al., 2013; Knights et al., 2013). It is recognized that an altered microbiome is not just a marker of disease but that it also actively contributes to pathogenesis (Chassaing et al., 2012). Reduced diversity of both fecal and mucosa-associated microbiota has been extensively reported in patients suffering from chronic intestinal inflammatory disease (Manichanh et al., 2006; Dicksved et al., 2008; Walker et al., 2011). Moreover, the composition of gut microbial communities may also modulate Type 1 diabetes (Wen et al., 2008).

Additionally, it is important to note that diet is one of the key factors affecting the balance of microbial populations in the digestive tract (Xu and Knight, 2014; Graf et al., 2015). Dietary nutrients are the principal substrates for the microbial population and also have a direct effect on the immune response (Faria et al., 2013). In laboratory mice, dietary-mediated effects are evident in a GI infection model (Ooi et al., 2014). Although the diet role in MAP infection has been scarcely studied, in previous experiments we observed that short term dietary shifts can modulate PTB infection in a rabbit model, showing differences in histopathological lesion extension and bacterial load on gut associated lymphoid tissue (GALT; Arrazuria et al., 2015a).

Rabbits are naturally susceptible to the development of MAP infections causing severe PTB-associated inflammatory responses in its wild habitats (Beard et al., 2001b; Maio et al., 2011), and milder pathological changes under experimental conditions (Vaughan et al., 2005; Arrazuria et al., 2015a). These characteristics, together with their size and handling easiness make rabbits a convenient experimental species to study chronic intestinal diseases of animals and humans. One specific feature of rabbit GALT is that it is composed of two additional special structures: the sacculus rotundus, which is located at the ileo-cecal junction, and the vermiform appendix, located at the end of the cecum. These two lymphoid organs account for more than $50 \%$ of the total lymphoid tissue in the rabbit (Rees Davies et al., 2003). These tissues can induce an effective immune response and it has been demonstrated that a specific microbial composition on the vermiform appendix can induce inflammation (Shanmugam et al., 2005). The sacculus rotundus closely resembles the ileocecal-valve in ruminants, and in previous MAP infection studies with rabbits we detected higher MAP loads in sacculus rotundus compared to vermiform appendix and any other sites in the intestine (Arrazuria et al., 2015a).

Recent advances in next generation sequencing technologies have enabled researchers to investigate the complete microbial composition of the GI tract under different conditions (Schuster, 2008). In the present study, high-throughput sequencing of the $16 \mathrm{~S}$ rRNA gene was performed to determine MAP infection associated shifts in the microbial composition of cecal content and sacculus rotundus.

Previous results from our group have shown that diet shifts can modulate MAP infection (Arrazuria et al., 2015a). In humans, high fiber diets have been shown to promote and increase the gut microbiota diversity (Steinle et al., 2013) and also to diminish inflammatory responses by a mechanism that includes shaping the intestinal microbiome and indirectly affecting the immune system (Kuo, 2013). On the basis of previously obtained results, in which a high fiber diet during challenge produced MAP infection modulation (Arrazuria et al., 2015a), we again performed this dietary shift to investigate if it produces changes in gut microbiota that could help understanding MAP infection modulation.

We hypothesized that as in other chronic intestinal diseases, MAP infection may produce changes in the gut microbiota. Therefore, our aim was to evaluate changes in gut microbiota in rabbits challenged with MAP and fed either a regular or a high fiber diet.

\section{MATERIALS AND METHODS}

\section{Animals and Experimental Designs}

New Zealand white female rabbits $(n=20)$ were purchased from authorized experimental animals dealers, Granja San Bernardo, Tulebras, Spain and arrived at NEIKER animal facilities, Derio, Spain, at the age of 6 weeks and $1.5 \mathrm{~kg}$ of body weight. After a 2-week adaptation period during which rabbits were fed special weaning pellets, all animals started taking a regular growing diet containing neither antibiotics nor coccidiostatics (Table $\mathbf{1}$ ). To prevent obesity, feeding was limited to $30-35$ g/day of Dry Matter/kg of live weight throughout the experiment. Two

TABLE 1 | The nutrient composition of the diets.

\begin{tabular}{lcc}
\hline Feed Composition & Regular diet & High fiber diet \\
\hline Dry Matter (\%) & 90.26 & 93.3 \\
Crude Protein (\%) & 16.32 & 2.69 \\
Crude Fat (\%) & 3.04 & 1.02 \\
Crude Fiber (\%) & 14.36 & 35.05 \\
Crude Ash (\%) & 7.04 & 8.40
\end{tabular}






different experiments were carried out. The experimental scheme is detailed on Figure 1.

In experiment 1 , in which all animals were fed a regular diet (Table 1), rabbits were split in two groups ( $n=5 /$ treatment); uninfected control (RC: Regular diet Control) and infected with MAP (RI: Regular diet Infected). The RI group was orally challenged 8 weeks after the beginning of the experiment (Figure 1) with $2 \mathrm{ml}$ of $2 \times 10^{8} \mathrm{cfu} / \mathrm{ml}$ of MAP strain K10 for three consecutive days as described previously (Arrazuria et al., 2015a).

In the second experiment, rabbits were also split in two groups ( $n=5 /$ treatment): (a) uninfected controls taking high fiber diet during the same period as their infected mates (HFC, high fiber control), and (b) twice infected with MAP taking high fiber diet during challenge (HFI, high fiber infected). In this experiment, regular diet was switched to a high fiber diet (Table 1) from week 7 to week 11 and from week 23 to week 26 in both groups (Figure 1) in order to modulate MAP infection as previously observed (Arrazuria et al., 2015a). The high fiber diet was switched back to the regular diet by replacing $25 \%$ of high fiber diet with regular diet weekly. The HFI group was orally challenged at week 8 and 24 with $2 \mathrm{ml}$ of $2 \times 10^{8} \mathrm{cfu} / \mathrm{ml}$ of MAP strain K10 for three consecutive days as described previously (Arrazuria et al., 2015a).

All four groups were housed in different rooms, with direct contact between animals belonging to the same group, and ad libitum water available on accessible containers fixed to the room walls. All animals were euthanized at week 28 of the experiment ( 36 weeks of age) by intracardiac pentobarbital injection after deep sedation with xylazine $(5 \mathrm{mg} / \mathrm{kg})$ and ketamine $(35 \mathrm{mg} / \mathrm{kg})$. Animals were exsanguinated and then necropsied.

This study was carried out following European, National, and Regional regulations on animals used in experimentation and other scientific purposes. The procedure was evaluated by the
Ethics Committee of the institution (NEIKER-OEBA-2014-0001) and authorized by the Regional Council (BFA-4269).

\section{Sampling, DNA Extraction, and PCR for MAP Detection}

The lymphoid tissues: sacculus rotundus, vermiform appendix, and mesenteric lymph node were excised and stored at $-20^{\circ} \mathrm{C}$ until further analyses.

DNA extraction was performed using DNA ExtractVK (Vacunek S.L, Bizkaia, Spain) following manufacturer's instructions as described previously (Arrazuria et al., 2015b). Briefly, DNA extraction was carried out on $300 \mathrm{mg}$ of tissue, with a combination of mechanical disruption of the cell walls using glass microspheres $(100 \mu \mathrm{m})$ agitation and a chemical lysis with SDS and proteinase K. DNA purification was achieved via silica based spin columns (Vacunek S.L, Bizkaia, Spain).

To identify MAP positive samples, DNA was subjected to a real-time multiplex PCR assay targeting the IS900 and ISMap02 DNA sequences of MAP (Sevilla et al., 2014). The reaction mixture contained 1x TaqMan Universal PCR Master Mix (Applied Biosystems, Madrid, Spain), 300-400 nM of each primer, $200 \mathrm{nM}$ of each probe, and $5 \mu \mathrm{l}$ of DNA extract in a final volume of $25 \mu$ l. Negative DNA extraction controls, nontemplate DNA and a positive PCR control were included in each PCR assay. A competitive internal amplification control (IAC) was also included in the PCR reaction. Amplification was carried out in a 7500 Real-Time PCR instrument (Applied Biosystems, Madrid, Spain) and consisted of one denaturation and polymerase activation cycle of $10 \mathrm{~min}$ at $95^{\circ} \mathrm{C}$, and 45 cycles of denaturation at $95^{\circ} \mathrm{C}$ for $15 \mathrm{~s}$ and annealing/extension at $60^{\circ} \mathrm{C}$ for $1 \mathrm{~min}$. Results were analyzed using the 7500 System SDS software v. 1.4 (Applied Biosystems). Threshold cycle $\left(C_{T}\right)$ and baseline were automatically determined by the software and verified by visual examination of the threshold line in amplification plots. $C_{T}$ values equal or below 38 for both IS900 
and ISMap02 probes were considered positive, $C_{T}$ values over 38 for both targets probe and under 38 for IAC probe were considered negative.

\section{Sampling and DNA Extraction for 165 rRNA Gene Sequencing}

To obtain the samples of cecal content, a small incision about $5 \mathrm{~cm}$ long was made in the middle of the cecum and approximately one gram of cecal content was taken and immediately saved in a sterile microtube. The sacculus rotundus was carefully excised and washed with sterile PBS to remove the digesta residues. A fragment was divided and saved immediately in a sterile microtube. All samples were stored in liquid nitrogen until further processing.

The frozen cecal content was defrosted and total DNA was extracted from $150 \mathrm{mg}$ of each sample using a ZR Fecal DNA MiniPrep (Zymo Research, Freiburg, Germany) according to manufacturer's instructions. This extraction kit includes a beadbeating step for the mechanical lysis of the cells.

The DNA extraction from the sacculus rotundus was carried out with Ultra-Deep Microbiome Prep kit (Molzym, Bremen, Germany) following manufacturer's instructions. This extraction method allows the enrichment of bacterial DNA and removal of animal and "dead" microbial DNA. Briefly, $0.25 \mathrm{~cm}^{2}$ of tissue sample was treated for host cell lysis under chaotropic conditions. The released DNA was then enzymatically degraded, and then the degrading enzymes inactivated. After bacterial cell wall degradation the DNA was extracted and purified via silica based spin columns. DNA concentration and purity of all samples were determined with ND-1000 spectrophotometer (Nanodrop, Wilmington, DE, USA) by measuring the $A_{260 / 280}$. DNA quality was also evaluated by gel electrophoresis after standard PCR using universal primers pAF ( $5^{\prime}$-AGA GTT TGA TCC TGG CTC AG-3') and 530R (5'-CCG CGG CKG CTG GCAC- $\left.3^{\prime}\right)$. DNA extracts were stored at $-20^{\circ} \mathrm{C}$ until they were processed.

\section{Library Construction and MiSeq Illumina Sequencing}

The V3-V4 region of $16 \mathrm{~S}$ rRNA gene was targeted for PCR amplification using a modified F338 and barcoded R806 primers (Caporaso et al., 2012) as described previously (Derakhshani et al., 2016b). Briefly, PCR reaction for each sample was performed in duplicate and contained $1.0 \mu \mathrm{l}$ of pre-normalized DNA $(20 \mathrm{ng} / \mu \mathrm{l}), 1.0 \mu \mathrm{l}$ of each forward and reverse primers $(10 \mu \mathrm{M}), 12 \mu \mathrm{l}$ HPLC grade water (Fisher Scientific, ON, Canada) and $10 \mu l 5$ Prime Hot MasterMix (5 Prime, Inc., Gaithersburg, MD, USA). Reactions consisted of an initial denaturing step at $94^{\circ} \mathrm{C}$ for $3 \mathrm{~min}$ followed by 30 amplification cycles at $94^{\circ} \mathrm{C}$ for $45 \mathrm{~s}, 62^{\circ} \mathrm{C}$ for $60 \mathrm{~s}$, and $72^{\circ} \mathrm{C}$ for $90 \mathrm{~s}$; finalized by an extension step at $72^{\circ} \mathrm{C}$ for $10 \mathrm{~min}$ in an Eppendorf Mastercycler pro (Eppendorf, Hamburg, Germany). PCR products were then purified using ZR-96 DNA Clean-up Kit (Zymo Research, Irvine, CA, USA) to remove primers, dNTPs and reaction components. The V3-V4 libraries were then generated by pooling $200 \mathrm{ng}$ of each sample, quantified by Picogreen dsDNA (Invitrogen,
New York, NY, USA) and diluted to a final concentration of 5 pM, measured by Qubit 2.0 Fluorometer (Life technologies, Ottawa, ON, Canada). In order to improve the unbalanced and biased base composition of the generated 16S rRNA libraries, $15 \%$ of PhiX control library was spiked into each amplicon pool. Customized sequencing primers for read1 (5'TATGGTAATTGTGTGCCAGCMGCCGCGGTAA-3'), $\operatorname{read} 2$ (5'-AGTCAGTCAGCCGGACTACHVGGGTWTCTAAT-3') and index read (5'-ATTAGAWACCCBDGTAGTCCGGCTGAC TGACT- $3^{\prime}$ ) were synthesized and purified by polyacrylamide gel electrophoresis (Integrated DNA Technologies, Coralville, IA, USA) and added to the MiSeq Reagent Kit V3 (600-cycle; Illumina, San Diego, CA, USA). The 300 paired-end sequencing reactions were performed on a MiSeq platform (Illumina, San Diego, CA, USA) at the Gut Microbiome and Large Animal Biosecurity Laboratories, Department of Animal Science, University of Manitoba, Canada. The sequencing data were deposited into the Sequence Read Archive (SRA) of NCBI (http://www.ncbi.nlm.nih.gov/sra) and can be accessed via accession number SRR2962702.

\section{Bioinformatics and Statistical Analyses}

The FLASH assembler (Magoč and Salzberg, 2011) was used to merge overlapping paired-end Illumina fastq files. All the sequences with mismatches or ambiguous calls in the overlapping region were discarded. The output fastq file was then analyzed by downstream computational pipelines of the open source software package QIIME version 1.9.0 (Caporaso et al., 2010b). Assembled reads were demultiplexed according to the barcode sequences and exposed to additional quality-filters so that reads with ambiguous calls and those with phred quality scores (Q-scores) below 20 were discarded. Chimeric reads were filtered using UCHIME (Edgar et al., 2011) and sequences were assigned to operational taxonomic units (OTU) using the QIIME implementation of UCLUST (Edgar, 2010) at 97\% pairwise identity threshold. Taxonomies assignment of representative OTUs and alignment to

TABLE 2 | Mycobacterium avium subspecies paratuberculosis (MAP) PCR results in the infected animals.

\begin{tabular}{lcccc}
\hline & & \multicolumn{3}{c}{ PCR results for indicated site } \\
\cline { 3 - 5 } Group & Animal ID & SR & VA & MLN \\
\hline $\mathrm{RI}$ & 16 & - & - & - \\
& 17 & + & - & - \\
& 18 & - & - & + \\
& 19 & - & - & + \\
& 20 & + & - & + \\
$\mathrm{HFI}$ & 11 & - & - & + \\
& 12 & + & + & + \\
13 & - & + & + \\
& 14 & - & - & + \\
15 & & - & & +
\end{tabular}

RI, regular diet infected; HFl, high fiber diet infected; SR, sacculus rotundus; VA, vermiform appendix; MLN, mesenteric lymph node. 


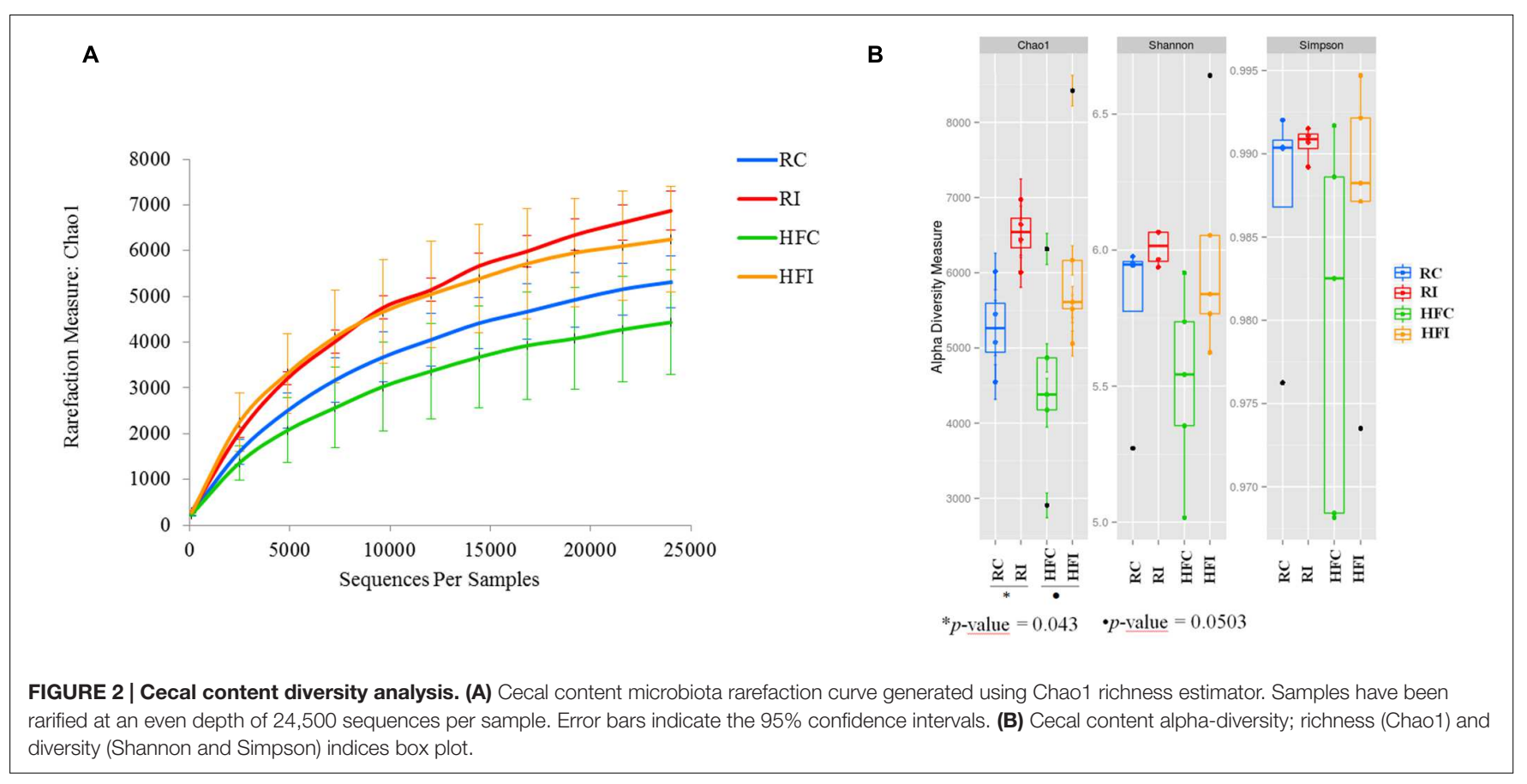

Silva reference database (Quast et al., 2013) were performed using PyNAST algorithms (Caporaso et al., 2010a). Phylogenetic trees were built with FastTree 2.1.3 for further comparisons between microbial communities (Price et al., 2010).

Within community diversity (alpha-diversity) was calculated using QIIME scripts. An even depth of 24,500 and 12,400 sequences per sample was used for calculation of species richness (Chao1; Chao, 1984) and diversity indices (Shannon and Simpson) for the cecal content and sacculus rotundus, respectively. Alpha rarefaction curves were generated using Chao1 estimator of species richness (Chao, 1984) with 10 sampling repetitions at each sampling depth. UNIVARIATE procedure of SAS (SAS 9.3, SAS Institute Inc., Cary, NC, USA) was used to test the normality of residuals for alphadiversity indices and the average of bacterial phyla. A logarithmic transformation was used to normalize the data when necessary. Comparisons between groups were performed using Student's $t$-test.

The diversity between animals and treatments (beta-diversity) was compared using weighted and unweighted UniFrac distances (Lozupone and Knight, 2005) based on phylogenetic differences. Principal coordinate analysis (PCoA) was applied on resulting distance matrices to generate two-dimensional plots using PRIMER V6 software (Warwick and Clarke, 2006) and permutational multivariate analysis of variance (PERMANOVA; Anderson, 2005) was used to calculate $p$-values and test for differences between microbial communities.

Microbial community between infected and non-infected animals was analyzed through orthogonal projection to latent structures discriminant analysis (OPLS-DA) using the SIMCA 14 software suite (Umetrics, Malmö, Sweden). The OPLS-DA is an extension of PLS-DA, featuring an integrated orthogonal signal correction (OSC) filter to remove variability not relevant to class

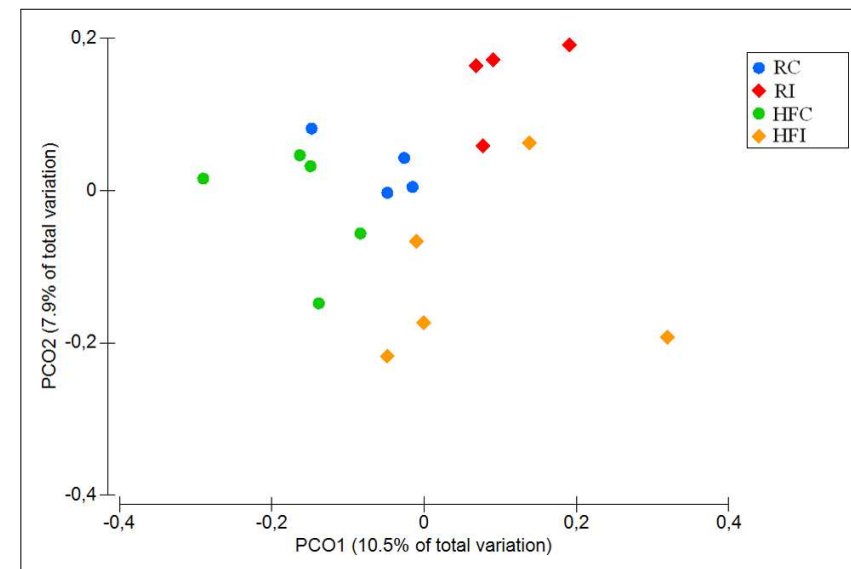

FIGURE 3 | Cecal content principal coordinates analysis (PCoA) of unweighted UniFrac distances. Significant $p$-value $=0.01$ for Infected vs. non-infected comparison was obtained from PERMANOVA.

separation (Trygg and Wold, 2002). In OPLS-DA a regression model is calculated between the multivariate data and a response variable that only contains class information. The OPLS-DA models were fitted with one predictive and two orthogonal components $(1+2)$. The quality of the models was evaluated with $R^{2} Y$ and $Q^{2}$, indicating the percent of variation of the training set explained by the $Y$-predictive components (infection status) and the cross-validated predicted variation, respectively. An $\mathrm{R}^{2} Y$ value close to 1 (explained variation) and $Q^{2}$ value larger than 0.5 is indicative of a good model with good predictability (Trygg and Wold, 2002).

For all analyses, a $p$-value of $<0.05$ was considered to be statistically significant and $<0.1$ was considered as a trend. 
TABLE 3 | Average percentages of bacteria phyla in the cecal content.

\begin{tabular}{|c|c|c|c|c|c|c|}
\hline \multirow[t]{3}{*}{ Phyla } & \multicolumn{4}{|c|}{ Mean values, \%, under indicated conditions (SEM) } & \multicolumn{2}{|c|}{$p$-values } \\
\hline & \multicolumn{4}{|c|}{ Treatments } & \multirow[t]{2}{*}{ RC vs. RI } & \multirow[t]{2}{*}{ HFC vs. HFI } \\
\hline & $\mathrm{RC}$ & $\mathbf{R I}$ & HFC & HFI & & \\
\hline Firmicutes & 71.01 (3.59) & $69.05(2.16)$ & $64.75(0.56)$ & $64.99(0.59)$ & 0.725 & 0.918 \\
\hline Bacteroidetes & $13.73(4.70)$ & $12.21(1.70)$ & $16.86(1.36)$ & $14.16(1.37)$ & 0.755 & 0.554 \\
\hline Cyanobacteria & $4.23(1.00)$ & $1.72(0.82)$ & 4.19 (1.03) & $5.18(1.08)$ & 0.118 & 0.731 \\
\hline Actinobacteria & $0.39(0.07)$ & $0.56(0.05)$ & $0.51(0.01)$ & $0.48(0.02)$ & $0.100^{2}$ & 0.817 \\
\hline Proteobacteria & $0.16(0.10)$ & $0.29(0.09)$ & $0.07(0.00)$ & $0.06(0.00)$ & 0.797 & $0.019^{1}$ \\
\hline TM7 & $0.16(0.03)$ & $0.26(0.04)$ & $0.47(0.02)$ & $0.16(0.02)$ & 0.853 & 0.147 \\
\hline Tenericutes & $0.43(0.13)$ & $0.16(0.05)$ & $0.34(0.06)$ & $0.78(0.07)$ & 0.955 & $0.052^{2}$ \\
\hline Unassigned & $9.89(0.93)$ & $15.75(2.61)$ & $12.81(1.04)$ & $14.18(1.42)$ & 0.132 & 0.732 \\
\hline
\end{tabular}

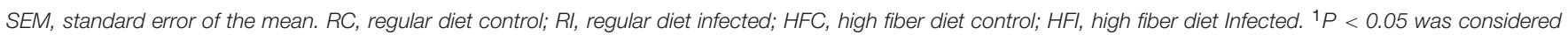
significant. ${ }^{2} P<0.1$ was discussed as trend.

\section{RESULTS}

\section{Determination of MAP Infection}

Mycobacterium avium subspecies paratuberculosis infection was confirmed following detection of MAP by PCR in at least one of the three lymphoid tissues (Table 2). All non-challenged animals yielded MAP negative PCR results, whereas all challenged group animals except one belonging to the RI group, were positive for at least one lymphoid tissue. Samples from the animal that rendered the negative PCR result were removed from all downstream analyses, and as such, only animals that presented at least one tissue positive in MAP PCR were included in analyses in their respective infected groups.

\section{Cecal Content Microbiota}

Following quality control and removal of chimeric reads, an average of 40,986 ( $S D=12,744)$ high quality sequences were obtained for cecal content samples and used for downstream analyses. Analyses of microbial communities revealed differences in richness (Chaol) between infected and control animals (Figure 2A). The RI animals presented higher richness values than RC (6873 \pm 481 vs. $5313 \pm 651 ; p=0.0043$; Figure 2B). An increasing trend was also observed in the Chaol richness estimates of HFI compared to HFC group $(6274 \pm 1299$ vs. $4434 \pm 1273 ; p=0.0503)$. However, the Shannon and Simpson indices were not significantly different between infected and control animals in any of the diet groups.

The beta-diversity analysis of unweighted UniFrac distances revealed distinct clustering pattern for the cecal content microbiota of MAP infected and control animals $(p=0.01$; Figure 3). However, when the weighted UniFrac distances were analyzed, no significant differences were observed between the microbiota composition of infected and non-infected animals $(p=0.2$; Supplementary Figure $\mathbf{S 1})$.

Alignment of OTUs at $97 \%$ similarity threshold against Silva database resulted in identification of seven bacterial phyla and 51 bacterial genera in the cecal content (Table 3, Figure 4). While majority of OTUs were identified at the genus level, some were only classified at the phylum, class, order, or family. The cecal content microbiota was dominated by members of phylum Firmicutes followed by members of phylum Bacteroidetes. Among all phyla, only the abundance of Proteobacteria significantly declined following MAP infection in animals fed high fiber diet $(p=0.019)$.

The OPLS-DA of cecal content bacteria for $Y$ variable (infection) yielded a suitable model for microbial comparison between infected and non-infected animals fed the regular $\left(R^{2} Y=1 ; Q^{2}=0.678\right)$ or the high fiber diets $\left(R^{2} Y=0.988\right.$; $Q^{2}=0.733$; Figure 4).

In RI animals most of the bacteria positively associated with MAP infection belonged to the phylum Firmicutes including family Dehalobacteriaceae, Mogibacteriaceae, Lachnospiraceae (including genera Anaerostipes and Moryella) and Eubacteriaceae (including genus Anaerofustis). In addition, family Coriobacteriaceae (phylum Actinobacteria) was more abundant in RI group than in RC (Figure 4A, Supplementary Figure S2A). On the other hand bacterial phyla showing negative association with MAP infection while positively related with RC group were more diversified including genus Lachnobacterium (phylum Firmicutes); family Enterobacteriaceae (phylum Proteobacteria); family S24-7 (phylum Bacteroidetes); orders YS2 and Streptophyta (phylum Cyanobacteria); order ML615J28 (phylum Tenericutes), and unclassified members of phylum Lentisphaerae (Figure 4A, Supplementary Figure S2A).

Animals fed the high fiber diet showed a different pattern of bacteria positively associated with MAP infection (Figure 4B, Supplementary Figure S2B). Within the phylum Firmicutes, several taxa including genera Ruminococcus, Clostridium, and Butyrivibrio, and family Erysipelotrichaceae were positively associated with HFI. In this group, members of phylum Proteobacteria were more abundant than in RI group. Examples included family Enterobacteriaceae (class Gammaproteobacteria); family Comamonadaceae; order Burkholderiales (class Betaproteobacteria); and genus Bilophila (class Deltaproteobacteria). Bacterial lineages belonging 


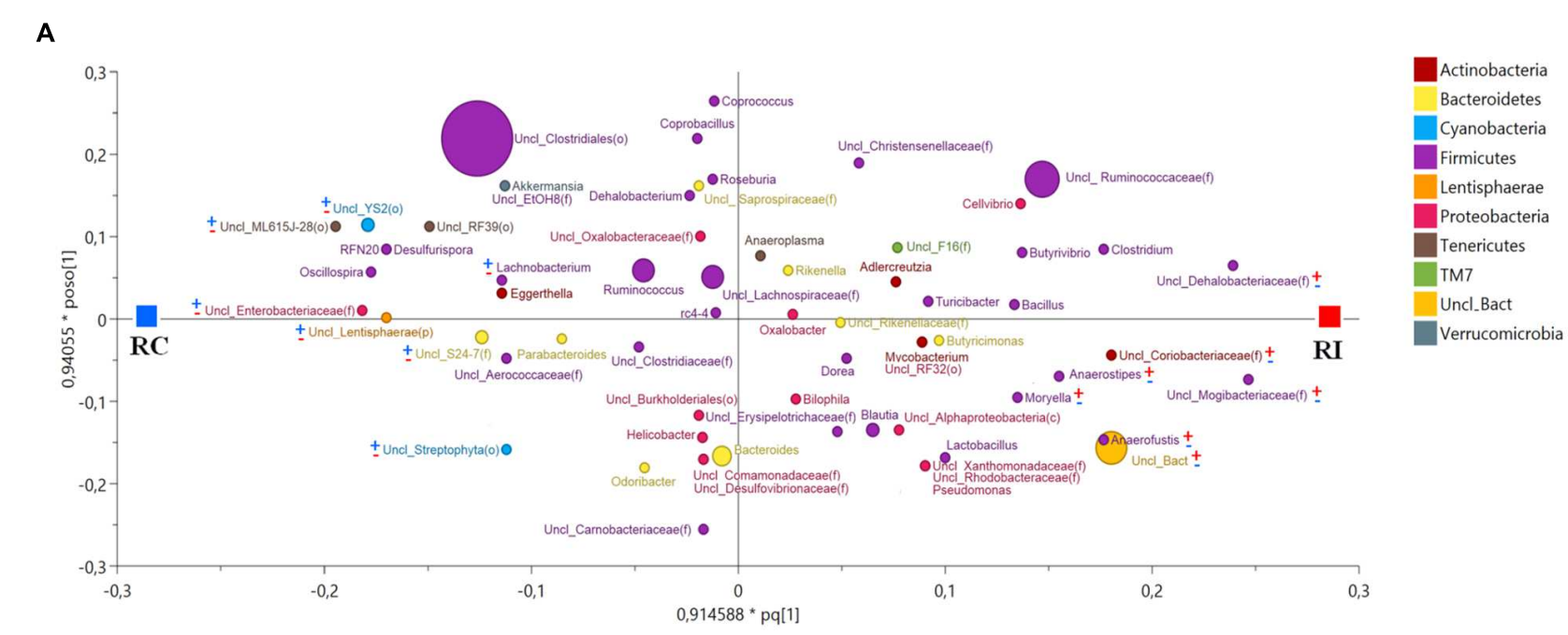

B

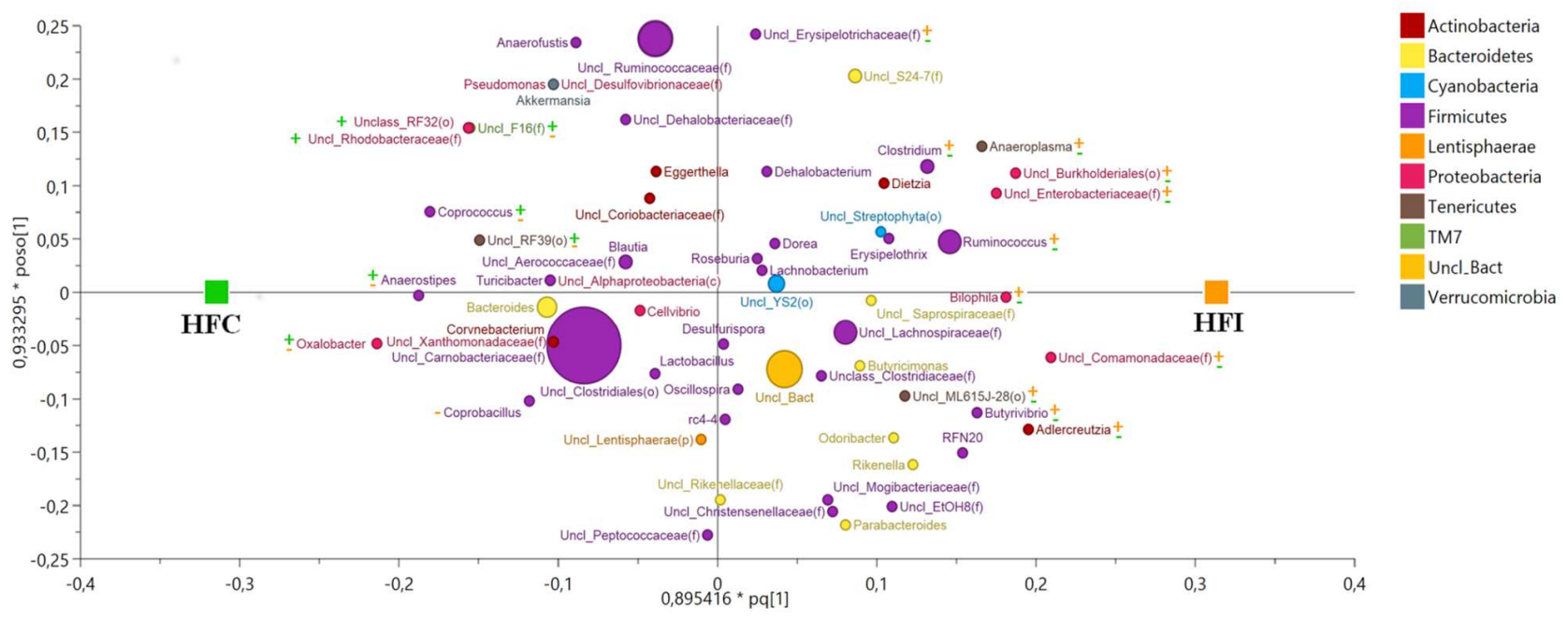

FIGURE 4 | Cecal content microbiota orthogonal projection to latent structures discriminant analysis (OPLS-DA) scatter plot. The size of the spheres represents abundance. (A) OPLS-DA Scatter Plot for cecal content samples of animals fed with regular diet $\left(R^{2} Y=1 ; Q^{2}=0.678\right)$. The taxa with significant positive association with MAP infected animals are labeled with $(+)$ and with negative association with MAP infected animal with (-). The taxa with significant positive association with control animals are labeled with $(+)$ and with negative association with control animals with (-). (B) OPLS-DA scatter plot for cecal content samples of animals fed with high fiber diet $\left(R^{2} Y=0.988 ; Q^{2} Y=0.733\right)$. The taxa with significant positive association with MAP infected animals are labeled with $(+)$ and with negative association with MAP infected animal with (-). The taxa with significant positive association with control animals are labeled with $(+)$ and with negative association with control animals with (-).

to the phylum Actinobacteria (genus Adlercreutzia) and phylum Tenericutes (genus Anaeroplasma and family ML615J28) were also more abundant in the HFI group compared to HFC.

The bacterial taxa showing negative association with MAP infection in animals fed high fiber diet and therefore more abundant in the HFC group, were different from those described in the RC group. These included genera Coprococcus, Anaerostipes, and Coprobacillus (phylum Firmicutes); genus Oxalobacter, family Rhodobacteraceae, and order RF32 (phylum Proteobacteria); order RF39 (phylum Tenericutes); and family F16 (phylum TM7; Figure 4B, Supplementary Figure S2B).

\section{Sacculus Rotundus Microbiota}

An average of $26,900(S D=10,284)$ of high quality sequences were obtained from sacculus rotundus samples. There were no significant differences in richness (Chaol index), or other alpha-diversity indices (Shannon and Simpson) when infected and non-infected animals were compared (Figure 5). The beta-diversity analysis of UniFrac distances revealed differences between infected and non-infected animals in both weighted 


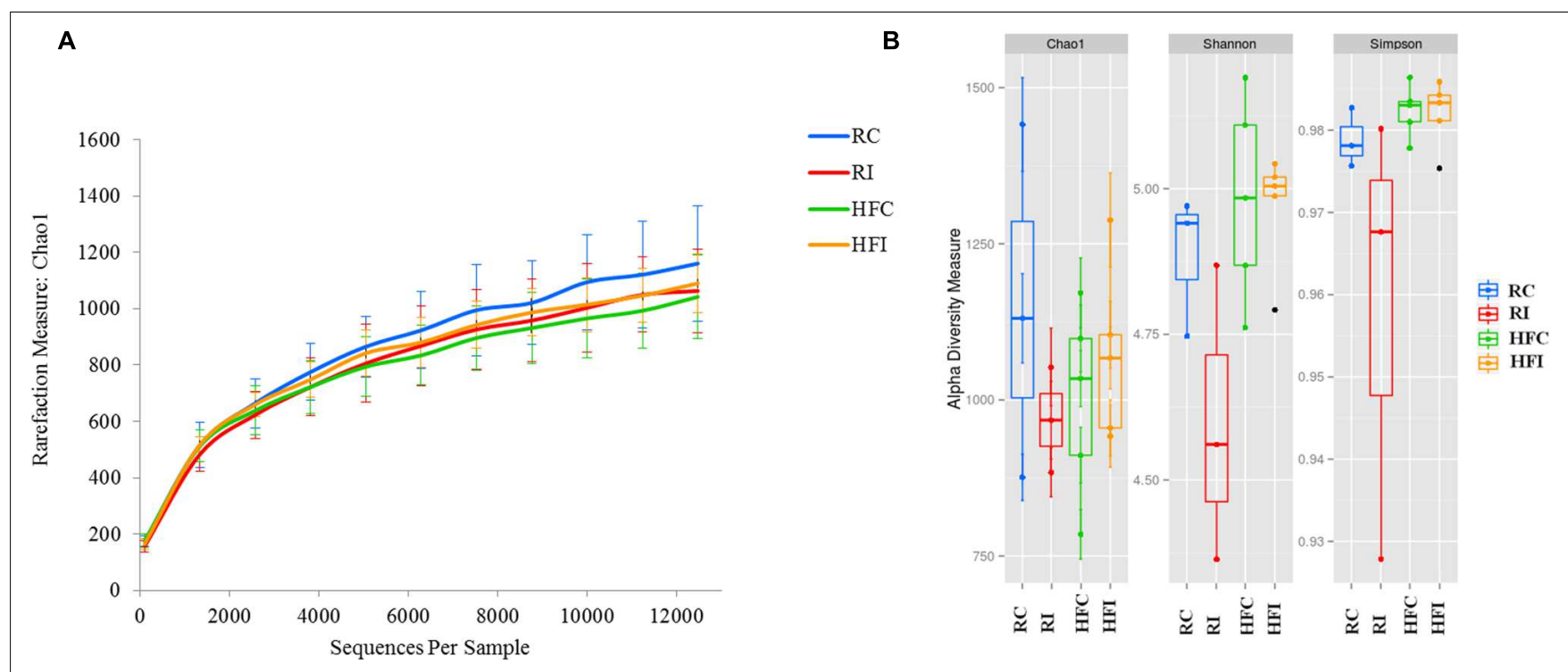

FIGURE 5 | Sacculus rotundus diversity analysis. (A) Sacculus rotundus rarefaction curve generated using Chao1 richness estimator. Samples have been rarified at an even depth of 12,400 sequences per sample. Error bars indicate the $95 \%$ confidence intervals. (B) Sacculus rotundus alpha-diversity; richness (Chao1) and diversity (Shannon and Simpson) indices box plot.

$(p=0.048)$ and weighted $(p=0.032)$ measures (Figures 6 and 7$)$. In this lymphoid tissue, 7 bacteria phyla and 63 bacteria genera were identified.

Similar to the cecal content, sacculus rotundus microbiota was mainly composed of phylum Firmicutes (Table 4). In contrast, phylum TM7 was the second most abundant phylum in the sacculus rotundus ranging from 2.3 to $5.9 \%$ of community, while its relative abundance ranged from 0.16 to $0.47 \%$ in the cecal content. The only phylum that significantly shifted with infection was Bacteroidetes, with declining abundance in MAP infected rabbits fed the regular diet. The composition of the OTUs at the genus level in the four groups is presented in Figure 8.

As in the cecal content, the OPLS-DA analysis of bacterial community in sacculus rotundus for $Y$-variable (infection) yielded a model with high goodness of fit $\left(R^{2} Y\right)$ and predicted value $\left(Q^{2}\right)$ both for animals fed with regular diet $\left(R^{2} Y=0.995\right.$, $\left.Q^{2}=0.765\right)$ and high fiber $\operatorname{diet}\left(R^{2} Y=0.921, Q^{2}=0.511\right.$; Figure 9).

Several bacterial taxa were positively associated with MAP infection and therefore more abundant in the RI compared to the RC group. This included the families Mogibacteriaceae, Dehalobacteriaceae, and Coriobacteriaceae and the genus Stenotrophomonas. A large number of taxa had negative association with MAP infection and therefore were overrepresented in the RC group comparing to RI one (Figure 9, Supplementary Figures S3A,B). Among these taxa, three also had negative association with infection in the cecal content and belonged to the phylum Tenericutes (order ML615J-28) and phylum Cyanobacteria (orders Streptophyta and YS2; Figure 9A, Supplementary Figure S3A).

Among animals fed the high fiber diet, genus Anaerofustis and family Lachnospiraceae were more abundant in the HFI group compared to the HFC one and therefore they were positively

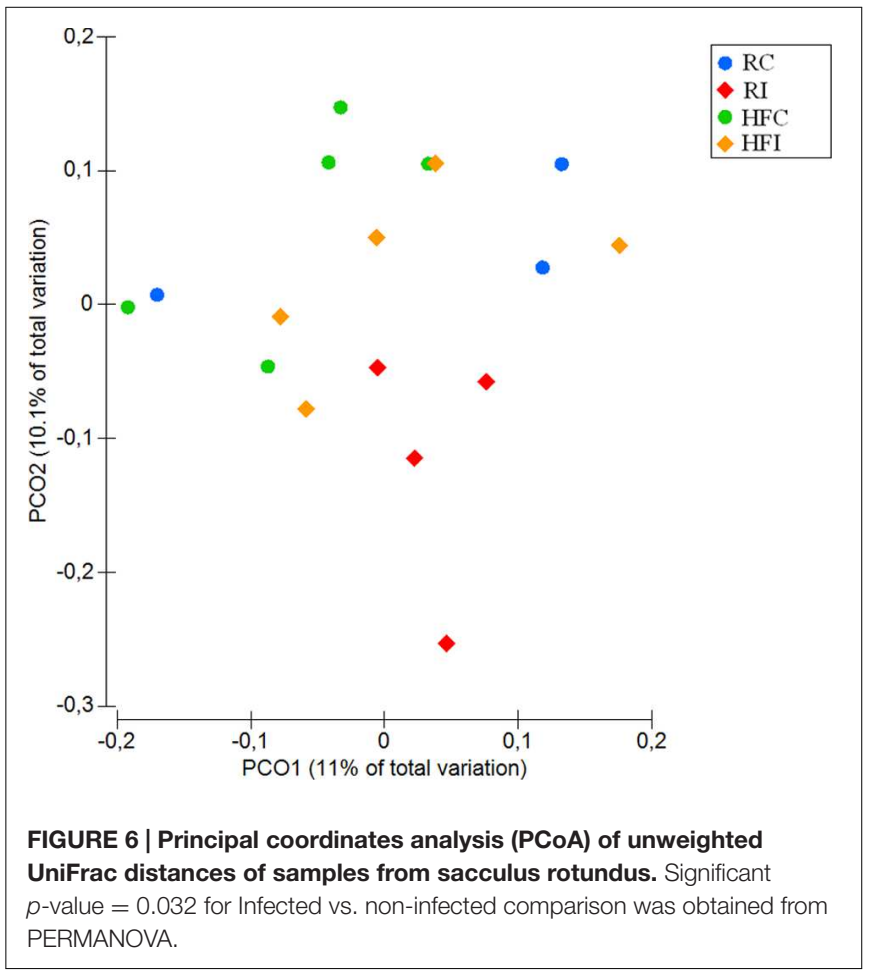

associated with infection. The two taxa with negative association with infection and therefore more abundant in the HFC group, belonged to the phylum Firmicutes, including families Erysipelotrichaceae (genus Coprobacillus) and Lachnospiraceae (genus Anaerostipes; Figure 9B, Supplementary Figure S3B). These two genera were also more abundant in the cecal content of HFC group and thus negatively associated with MAP infection. 
TABLE 4 | Average percentage of bacteria phyla in the sacculus rotundus samples.

\begin{tabular}{|c|c|c|c|c|c|c|}
\hline \multirow[t]{3}{*}{ Phyla } & \multicolumn{4}{|c|}{ Mean values, \%, under indicated conditions (SEM) } & \multicolumn{2}{|c|}{$p$-values } \\
\hline & \multicolumn{4}{|c|}{ Treatments } & \multirow[t]{2}{*}{ RC vs. RI } & \multirow[t]{2}{*}{ HFC vs. HFI } \\
\hline & $\mathrm{RC}$ & $\mathbf{R I}$ & HFC & HFI & & \\
\hline Firmicutes & $89.28(5.37)$ & $89.55(1.61)$ & 81.78 (3.56) & $87.86(1.80)$ & 0.414 & 0.178 \\
\hline TM7 & $2.71(1.74)$ & $4.27(1.19)$ & $5.97(1.65)$ & $2.30(0.69)$ & 0.821 & $0.065^{2}$ \\
\hline Bacteroidetes & $2.13(0.11)$ & $1.49(0.25)$ & $0.93(0.27)$ & $1.75(0.75)$ & $0.014^{1}$ & 0.261 \\
\hline Actinobacteria & $0.95(0.35)$ & $2.14(0.64)$ & $1.17(0.20)$ & $0.99(0.19)$ & 0.150 & 0.378 \\
\hline Tenericutes & $0.83(1.11)$ & $0.27(0.09)$ & $0.95(0.39)$ & $0.79(0.20)$ & 0.398 & 0.504 \\
\hline Cyanobacteria & $0.66(1.11)$ & $0.20(0.10)$ & $5.14(1.95)$ & $1.36(0.54)$ & 0.467 & 0.212 \\
\hline Proteobacteria & $0.49(0.64)$ & $0.17(0.03)$ & $0.40(0.15)$ & $0.17(0.04)$ & 0.428 & 0.136 \\
\hline Unassigned & $2.97(0.82)$ & $1.91(0.08)$ & $3.66(0.60)$ & $4.76(1.16)$ & 0.242 & 0.620 \\
\hline
\end{tabular}

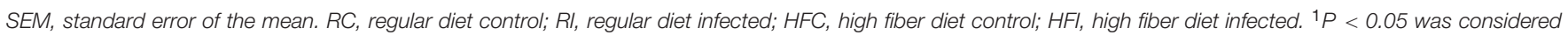
significant. ${ }^{2} P<0.1$ was discussed as trend.

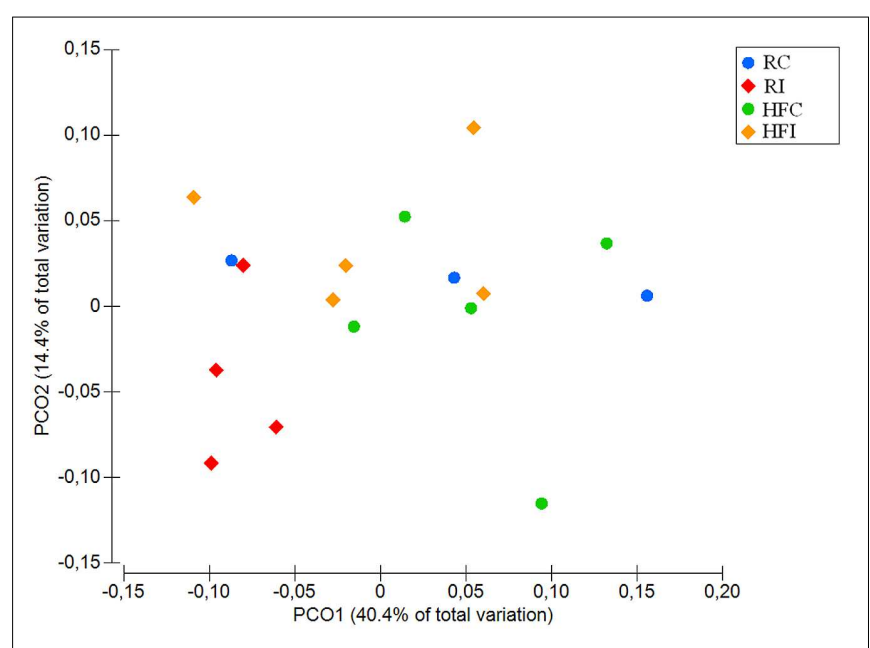

FIGURE 7 | Principal coordinates analysis (PCoA) of weighted UniFrac distances of samples from sacculus rotundus. Significant

$p$-value $=0.048$ for Infected vs. non-infected comparison was obtained from PERMANOVA.

\section{DISCUSSION}

In the present work, we attempt to study the impact of MAP infection on gut microbiota composition under two different dietary scenarios. Variations in the microbial community were observed depending on the dietary group and in response to MAP infection.

Cecal microbial community of rabbit has been assessed in recent studies (Bäuerl et al., 2014; Zhu et al., 2015) but to the best of our knowledge, this is the first work describing the microbiota of rabbit sacculus rotundus. This organ unique to rabbits is characterized by an ampoule-like enlargement of the terminal ileum. Due to its location and lymphoid nature it might play a similar role to the ileocecal-valve in ruminants (Besoluk et al., 2006) and can be assumed to bear microbiota with an important role in immune regulation.
Studies using high-throughput sequencing technology to explore the gut microbiota in rabbits are not abundant. These limited studies indicate that GI tract of rabbits is a highly diverse environment (Bäuerl et al., 2014; Zhu et al., 2015). In the present study, we observed that even with an average sequencing depth of $24,700 \mathrm{reads} / \mathrm{sample}$ in the cecal content and $12,400 \mathrm{reads} / \mathrm{sample}$ in the sacculus rotundus, rarefaction curves did not reach a plateau phase indicating that the ecosystem is highly diverse. These findings further support previous observations on high microbiota diversity in rabbit cecal content (Bäuerl et al., 2014; Zhu et al., 2015) and feces (Eshar and Weese, 2014; Zeng et al., 2015).

Microbiota richness, evenness and diversity have been reported as characteristic features determining state of health or disease. A decreased in richness and diversity indices has been reported in CD (Manichanh et al., 2006; Dicksved et al., 2008; Walker et al., 2011) and Type 1 diabetes patients (Giongo et al., 2011), whereas bacterial vaginosis (Ling et al., 2010) and helminthic parasitosis (Lee et al., 2014) have been associated with increased richness. In our study, the alpha-diversity indices in sacculus rotundus microbiota, did not differ between infected and non-infected animals. However, in the cecal content, we found higher values of richness (chaol index) in infected animals whereas the abundance-based diversity measures (Shannon and Simpson indices) did not vary between infected and non-infected animals. It must be taken into account that cecal content DNA extraction did not allow removing DNA from dead bacteria whereas, the extraction method used for sacculus rotundus did, and this could have influenced results.

The diversity analyses between animals (beta-diversity) revealed that the microbial composition between infected and non-infected animals varied in the cecal content and sacculus rotundus in response to MAP infection. In the cecal content, the significant differences were observed only in unweighted UniFrac distances, suggesting that MAP infection has an impact on the presence and/or absence of certain members of the microbial community rather than on the abundance of these. Due to rabbits' practice of cecotrophy, the composition of the microbial community of the cecal content may play a role in the modulation 


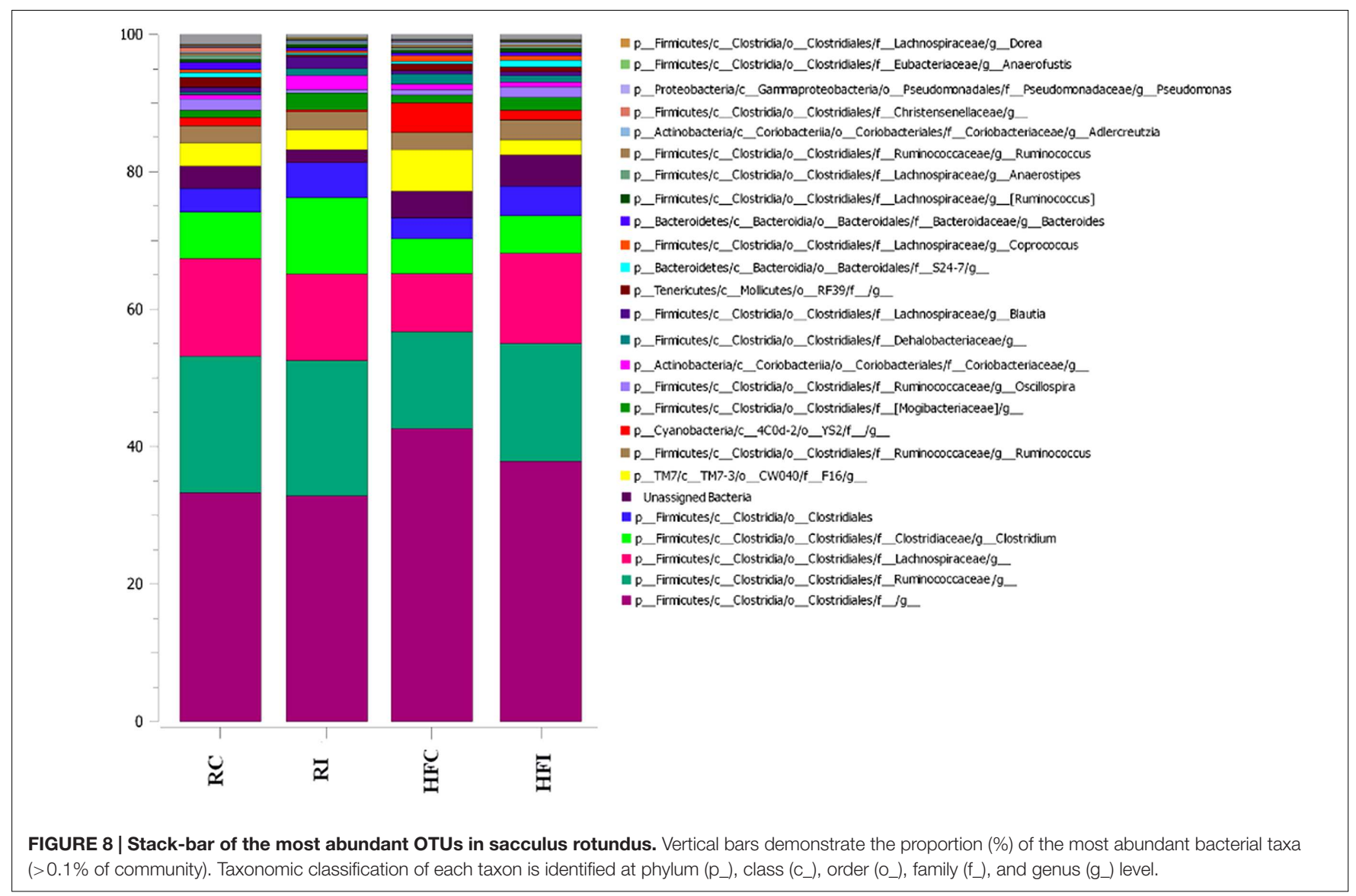

of nutrient absorption. In contrast, in sacculus rotundus, we observed changes in beta-diversity using both weighted and unweighted UniFrac distances, suggesting that MAP infection both impacted the presence and/or absence of certain taxa as well as their abundance. These observed changes may play a role in inflammation as demonstrated in previous reports where specific microbial compositions in rabbit's lymphoid tissue have been seen to induce inflammation (Shanmugam et al., 2005).

It was not surprising that rabbit cecal content was dominated by the phylum Firmicutes as reported in recent studies (Bäuerl et al., 2014; Zhu et al., 2015). This phylum contains some of the major cellulolytic and fibrolytic organisms (Daly et al., 2001) that can be also found in other hindgut fermenters, such as horses (Costa et al., 2012).

At the phylum level, we only observed significant differences in the phyla Proteobacteria and Bacteroidetes. The phylum Proteobacteria decreased in the cecal content of HFI group compared with HFC. However, in the animals fed with high fiber diet there were more bacteria belonging to this phylum positively associated with MAP infection than in animals fed with regular diet. Proteobacteria are considered to be minor and opportunistic components of the gut ecosystem. The proportion of this phylum increases in both IBD patients (Gophna et al., 2006) and animal models of IBD (Munyaka et al., 2016) suggesting these bacteria may play a role in pathogenesis of IBD (Mukhopadhya et al., 2012).
In our study, the phylum Bacteroidetes was decreased in the sacculus rotundus of RI compared with RC. This phylum is reported to decrease in experimentally MAP-infected calves (Derakhshani et al., 2016a) and in CD patients (Gophna et al., 2006). The phylum Bacteroidetes has a major role in degrading complex polysaccharides (Terrapon and Henrissat, 2014) that are normally present in high fiber diets. Studies have shown that high fiber based diets promote the abundance of Bacteroidetes in rabbits gut (Nicodemus et al., 2005; Zhu et al., 2015). In our previous study, we reported that a shift to a high fiber diet during MAP challenge may decelerate the progression of infection (Arrazuria et al., 2015a). The increase of Bacteroidetes may be in part responsible for this finding.

Genus Mycobacterium was only detected in one cecal content sample from an animal belonging to the infected group fed the regular diet (Figure 4A, Supplementary Figure S2A). Since the classification was at the genus level we cannot confirm that it is MAP. In slaughtered rabbits, detection of other members of Mycobacterium avium complex has been reported in lymphoid tissue, although no mycobacteria were found in the cecal content (Arrazuria et al., 2015b). The differences in MAP detection in sacculus rotundus by PCR (Arrazuria et al., 2015b) and sequencing could be due to the standardization of amount of DNA for sequencing, the choice of primers used (universal bacterial primers in case of sequencing vs. MAP 


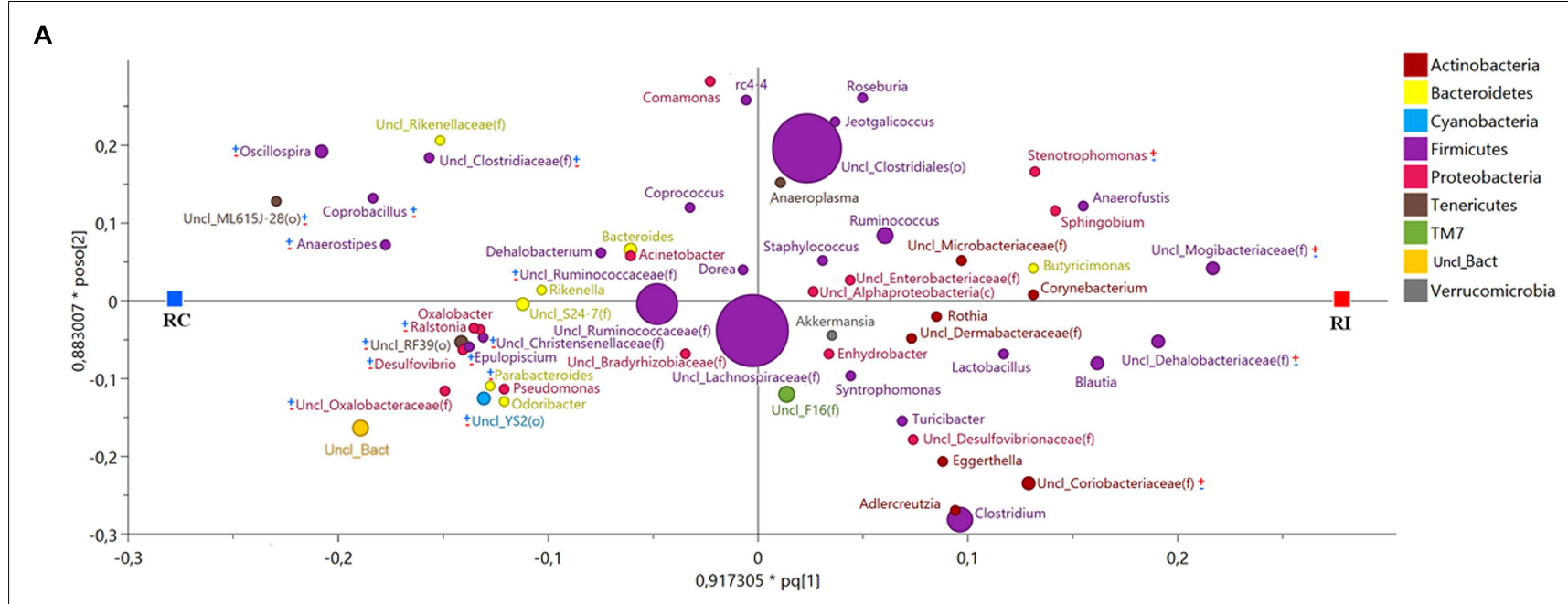

B

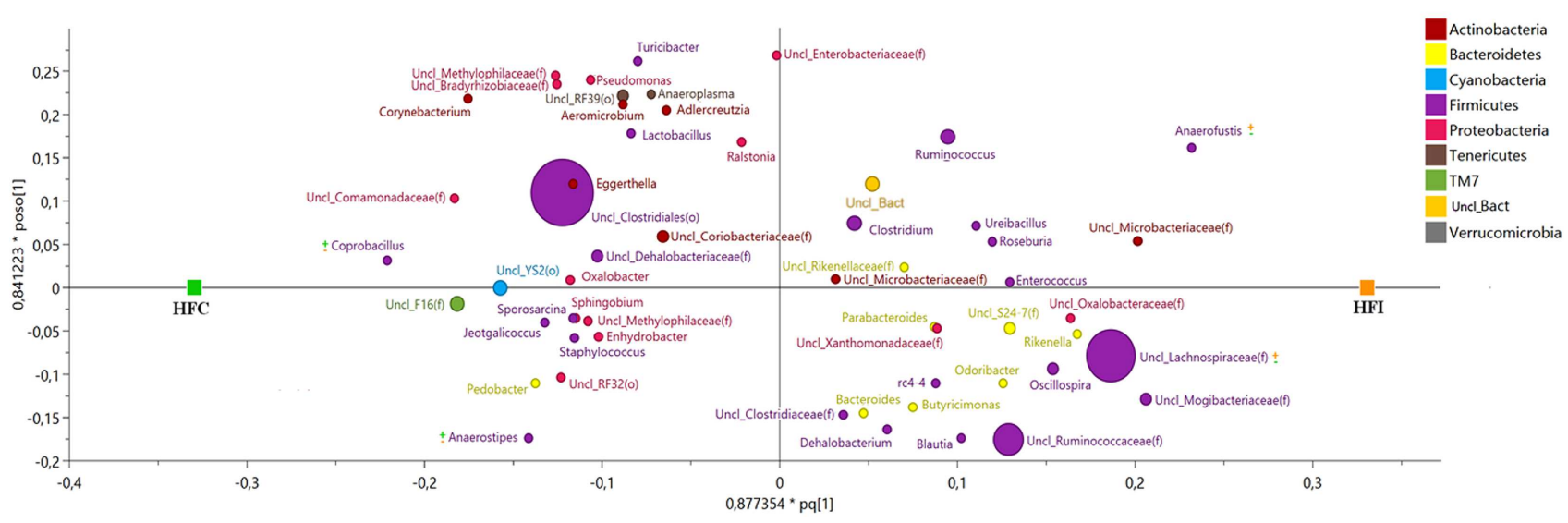

FIGURE 9 | Sacculus rotundus microbiota orthogonal projection to latent structures discriminant analysis (OPLS-DA) scatter Plot. The size of the spheres represents abundance. (A) OPLS-DA scatter plot for sacculus rotundus samples of animals fed with regular diet $\left(R^{2} Y=0.995 ; Q^{2}=0.765\right)$. The taxa with significant positive association with MAP infected animals are labeled with (+) and with negative association with MAP infected animal with (-). The taxa with significant positive association with control animals are labeled with $(+)$ and with negative association with control animals with (-). (B) OPLS-DA scatter plot for sacculus rotundus samples of animals fed with high fiber diet $\left(R^{2} Y=0.921 ; Q^{2}=0.511\right)$. The taxa with significant positive association with MAP infected animals are labeled with $(+)$ and with negative association with MAP infected animal with (-). The taxa with significant positive association with control animals are labeled with $(+)$ and with negative association with control animals with (-).

specific primers for PCR) or because the relative abundance of MAP in infection is not too high since the disease is of chronic nature.

We used an OPLS-DA model to determine which bacteria were positively or negatively associated with MAP infection. Using this approach, we observed that three families, Coriobacteriaceae, Dehalobacteriaceae, and Mogibacteriaceae were positively associated with MAP infection in both sacculus rotundus and the cecal content of the RI group. Some members of the family Coriobacteriaceae have been found in vaginosis, bacteriemia, and periodontitis, being considered pathobionts (Clavel et al., 2014). In the context of PTB, the increase of this family of pathobionts may have an implication in disease progression. Derakhshani et al. (2016a) reported that the family Mogibacteriaceae was the main overrepresented bacteria in the ileum mucosa of MAP infected calves. Unfortunately there is a lack of knowledge on the role that this family could play in gut inflammation.

The genus Anaerostipes was found to be more abundant in the cecal content of the RI group. It was also more abundant in sacculus rotundus of the RC and in cecal content and sacculus rotundus of HFC animals. The genus Anaerostipes can utilize lactate to produce butyrate (Sato et al., 2008), which can suppress pro-inflammatory cytokine production by intestinal macrophages (Chang et al., 2014) and it is beneficial to colonic health providing protection against colitis (Segain et al., 2000). Also, this genus was found in significant lower abundance in smoker CD patients compared to non-smokers (Morgan et al., 2012). These findings suggest that this genus may play an important role in GI health. 
The genus Stenotrophomonas was overrepresented in sacculus rotundus of $\mathrm{RI}$. It has been described in $\mathrm{CD}$, and has been recognized in many clinical specimens and inflammatory conditions like pneumonia, bacteremia, and urinary tract infection (Denton and Kerr, 1998; Knösel et al., 2009). This species also appears to be increased in mice females infected with MAP (Karunasena et al., 2014). These findings are in agreement with our results.

The genus Coprobacillus was more abundant in the control groups (sacculus rotundus of RC and in sacculus rotundus and cecal content of HFC). This genus has been found to be beneficial through maintaining intestinal stability and conferring resistance against Clostridium difficile colonization (Stein et al., 2013).

In the present study, sacculus rotundus and cecal content of the RC group showed higher levels of two orders that belong to the phylum Cyanobacteria (orders Streptophyta and YS2). Since Cyanobacteria has been detected in the GI tract of human and other mammals (Ley et al., 2008) the efforts to better characterize the function of members of this phylum are increasing (Rienzi et al., 2013; Soo et al., 2014). Streptophyta, which derive from chloroplasts within plant matter has been detected in high proportion in stool from a patient with resistant tuberculosis treated with broad-spectrum antibiotics (Dubourg et al., 2013), suggesting that the critical reduction of bacterial sequences detected had permitted the amplification of DNA from green plant foods absorbed by the patient. A recent genomic study showed that YS2 does not have photosynthetic ability. Metabolic analysis demonstrated that YS2 has many special functions including obligate anaerobic fermentation, syntrophic H2-production, nitrogen fixation, and synthesis of vitamin B and $\mathrm{K}$ (Rienzi et al., 2013). The infection may lead to the establishment of an ecosystem that is not favorable for this order and it could support the pathogenesis of the disease.

As shown in Figures 4 and 9, we found additional taxa that show positive or negative relation with MAP infection only under specific dietary regimens. Diets containing different amounts of fiber are likely to differentially modulate the composition of the intestinal microbiota (Serino et al., 2011; Zhu et al., 2015). We cannot rule out that some of the detected microbes were taken up with the diet. DNA from food adsorbed by the host has been reported as mentioned previously (Dubourg et al., 2013). Microbes taken up with food may succeed to survive, especially in the high fiber diet groups where the diet shift itself could have favored their survival. Finally, we cannot, speculate if the associated taxa with MAP-infection, only observed in animals fed the high fiber diet, are consequence of the short diet change, or on the contrary, are the result of MAP reinfection.

\section{CONCLUSION}

The MAP infection and dietary changes shift microbiota of the cecal content and the sacculus rotundus of rabbits. While some taxa seem to be positively associated with MAP infection in different sites or conditions, there are others that show opposite patterns. Knowing the composition of the microbial community alone does not lead to an understanding of its function. Therefore, it would be helpful to confirm and complete the results presented in the present study with a shotgun metagenomic or metatranscriptomics approach that could help to better understand the role of microbiota composition and function. Further research is required to understand the pathogenesis of MAP associated to chronic intestinal inflammation and the role that intestinal microbiota plays during this process.

\section{AUTHOR CONTRIBUTIONS}

RJ and NE conceived and designed the experiment. RA and NE conducted the animal experiment. RA and HD performed lab analyses. RA, HD, and EK developed the bioinformatics and statistical models. RA and HD analyzed the data. All authors drafted the manuscript. All authors carefully read and approved the final version of the manuscript.

\section{FUNDING}

The research was funded by grant (AGL2012-39818-C02-02) from the Spanish Ministry of Economy and Competitiveness (MINECO) and University of Manitoba Start-up fund. RA held a predoctoral fellowship (BFI-2012-237) and a visiting fellowship (EP_2015_1_53) from the Department of Education, Universities and Research of the Basque Government.

\section{SUPPLEMENTARY MATERIAL}

The Supplementary Material for this article can be found online at: http://journal.frontiersin.org/article/10.3389/fmicb. 2016.00446

FIGURE S1 | Cecal content principal coordinates analysis (PCoA) of weighted UniFrac distances. PERMANOVA analysis showed no significant differences between Infected vs. non-infected animals $p$-value $=0.2$.

FIGURE S2 | Cecal content OPLS-DA coefficient plot; bars with negative values indicating bacteria that are significantly lower in MAP infected animals and bars with positive value indicating bacteria which are significantly higher in MAP infected animals than in control animals. Only bacteria with a confidence interval that did not cross the zero line were significantly changed $* p<0.05$. (A) OPLS-DA coefficient plot for samples of sacculus rotundus from animals infected and fed with regular diet $\left(R^{2} Y=1 ; Q^{2}=0.678\right)$. (B) OPLS-DA coefficient plot for samples of sacculus rotundus from animals infected and fed high fiber diet $\left(R^{2} Y=0.988 ; Q^{2} Y=0.733\right)$.

\begin{abstract}
FIGURE S3 | Sacculus rotundus OPLS-DA coefficient plot; bars with negative values indicating bacteria that are significantly lower in MAP infected animals and bars with positive value indicating bacteria which are significantly higher in MAP infected animals than in control animals. Only bacteria with a confidence interval that did not cross the zero line were significantly changed ${ }^{*} p<0.05$. (A) OPLS-DA coefficient plot for samples of sacculus rotundus from animals infected and fed with regular diet $\left(R^{2} Y=0.995\right.$; $\left.Q^{2}=0.765\right)$. (B) OPLS-DA coefficient plot for samples of sacculus rotundus from animals infected and fed high fiber diet $\left(R^{2} Y=0.921 ; Q^{2}=0.511\right)$.
\end{abstract}




\section{REFERENCES}

Anderson, M. J. (2005). PERMANOVA: a FORTRAN Computer Program for Permutational Multivariate Analysis of Variance, thesis, Department of Static University Auckland, Auckland.

Arrazuria, R., Molina, E., Mateo-Abad, M., Arostegui, I., Garrido, J. M., Juste, R. A., et al. (2015a). Effect of various dietary regimens on oral challenge with Mycobacterium avium subsp. paratuberculosis in a rabbit model. Res. Vet. Sci. 101, 80-83. doi: 10.1016/j.rvsc.2015.06.006

Arrazuria, R., Sevilla, I. A., Molina, E., Pérez, V., Garrido, J. M., Juste, R. A., et al. (2015b). Detection of Mycobacterium avium subspecies in the gut associated lymphoid tissue of slaughtered rabbits. BMC Vet. Res. 11:130. doi: 10.1186/s12917-015-0445-2

Bäuerl, C., Collado, M. C., Zúñiga, M., Blas, E., and Pérez Martínez, G. (2014). Changes in cecal microbiota and mucosal gene expression revealed new aspects of epizootic rabbit enteropathy. PLOS ONE 9:e105707. doi: 10.1371/journal.pone.0105707

Beard, P. M., Daniels, M. J., Henderson, D., Pirie, A., Rudge, K., Buxton, D., et al. (2001a). Paratuberculosis infection of nonruminant wildlife in Scotland. J. Clin. Microbiol. 39, 1517-1521. doi: 10.1128/JCM.39.4.1517-1521.2001

Beard, P. M., Rhind, S. M., Buxton, D., Daniels, M. J., Henderson, D., Pirie, A., et al. (2001b). Natural paratuberculosis infection in rabbits in Scotland. J. Comp. Pathol. 124, 290-299. doi: 10.1053/jcpa.2001.0466

Besoluk, K., Eken, E., and Sur, E. (2006). A morphological and morphometrical study on the sacculus rotundus and ileum of the Angora rabbit. Veterin. Med. Praha 51, 60.

Caporaso, J. G., Bittinger, K., Bushman, F. D., DeSantis, T. Z., Andersen, G. L., and Knight, R. (2010a). PyNAST: a flexible tool for aligning sequences to a template alignment. Bioinformatics 26, 266-267. doi: 10.1093/bioinformatics/btp636

Caporaso, J. G., Kuczynski, J., Stombaugh, J., Bittinger, K., Bushman, F. D., Costello, E. K., et al. (2010b). QIIME allows analysis of highthroughput community sequencing data. Nat. Methods 7, 335-336. doi: 10.1038/nmeth.f.303

Caporaso, J. G., Lauber, C. L., Walters, W. A., Berg-Lyons, D., Huntley, J., Fierer, N., et al. (2012). Ultra-high-throughput microbial community analysis on the Illumina HiSeq and MiSeq platforms. ISME J. 6, 1621-1624. doi: 10.1038/ismej.2012.8

Chang, P. V., Hao, L., Offermanns, S., and Medzhitov, R. (2014). The microbial metabolite butyrate regulates intestinal macrophage function via histone deacetylase inhibition. Proc. Natl. Acad. Sci. U.S.A. 111, 2247-2252. doi: 10.1073/pnas.1322269111

Chao, A. (1984). Nonparametric estimation of the number of classes in a population. Scand. J. Stat. 11, 265-270.

Chassaing, B., Aitken, J. D., Gewirtz, A. T., and Vijay-Kumar, M. (2012). Gut microbiota drives metabolic disease in immunologically altered mice. $A d v$. Immunol. 116, 93-112. doi: 10.1016/B978-0-12-394300-2.00003-X

Chiodini, R. J., and Van Kruiningen, H. J. (1983). Eastern white-tailed deer as a reservoir of ruminant paratuberculosis. J. Am. Vet. Med. Assoc. 182, 168-169.

Clarke, C. J. (1997). The pathology and pathogenesis of paratuberculosis in ruminants and other species. J. Comp. Pathol. 116, 217-261. doi: 10.1016/S00219975(97)80001-1

Clavel, T., Lepage, P., and Charrier, C. (2014). The family coriobacteriaceae. Prokaryotes 49, 201-238.

Clemente, J. C., Ursell, L. K., Parfrey, L. W., and Knight, R. (2012). The impact of the gut microbiota on human health: an integrative view. Cell 148, 1258-1270. doi: 10.1016/j.cell.2012.01.035

Costa, M. C., Arroyo, L. G., Allen-Vercoe, E., Stämpfli, H. R., Kim, P. T., Sturgeon, A., et al. (2012). Comparison of the fecal microbiota of healthy horses and horses with colitis by high throughput sequencing of the V3-V5 region of the 16S rRNA gene. PLoS ONE 7:e41484. doi: 10.1371/journal.pone.0041484

Daly, K., Stewart, C. S., Flint, H. J., and Shirazi-Beechey, S. P. (2001). Bacterial diversity within the equine large intestine as revealed by molecular analysis of cloned 16S rRNA genes. FEMS Microbiol. Ecol. 38, 141-151. doi: 10.1111/j.1574-6941.2001.tb00892.x

Denton, M., and Kerr, K. G. (1998). Microbiological and clinical aspects of infection associated with Stenotrophomonas maltophilia. Clin. Microbiol. Rev. 11, 57-80.

Derakhshani, H., De Buck, J., Mortier, R., Barkema, H. W., Krause, D. O., and Khafipour, E. (2016a). The features of fecal and ileal mucosa-associated microbiota in dairy calves during early infection with Mycobacterium avium subspecies paratuberculosis. Front. Microbiol. 7:426. doi: 10.3389/fmicb.2016.00426

Derakhshani, H., Tun, H. M., and Khafipour, E. (2016b). An extended single-index multiplexed 16S rRNA sequencing for microbial community analysis on MiSeq illumina platforms. J. Basic Microbiol. 56, 321-326. doi: 10.1002/jobm. 201500420

Dicksved, J., Halfvarson, J., Rosenquist, M., Järnerot, G., Tysk, C., Apajalahti, J., et al. (2008). Molecular analysis of the gut microbiota of identical twins with Crohn's disease. ISME J. 2, 716-727. doi: 10.1038/ismej.2008.37

Dubourg, G., Lagier, J. C., Armougom, F., Robert, C., Hamad, I., Brouqui, P., et al. (2013). The gut microbiota of a patient with resistant tuberculosis is more comprehensively studied by culturomics than by metagenomics. Eur. J. Clin. Microbiol. Infect. Dis. 32, 637-645. doi: 10.1007/s10096-012-1787-3

Edgar, R. (2010). Search and clustering orders of magnitude faster than BLAST. Bioinformatics 26, 2460-2461. doi: 10.1093/bioinformatics/btq461

Edgar, R., Haas, B., Clemente, J., Quince, C., and Knight, R. (2011). UCHIME improves sensitivity and speed of chimera detection. Bioinformatics 27, 21942200. doi: 10.1093/bioinformatics/btr381

Eshar, D., and Weese, J. S. (2014). Molecular analysis of the microbiota in hard feces from healthy rabbits (Oryctolagus cuniculus) medicated with long term oral meloxicam. BMC Vet. Res. 10:62. doi: 10.1186/1746-6148-10-62

Faria, A. M. C., Gomes-Santos, A. C., Gonçalves, J. L., Moreira, T. G., Medeiros, S. R., Dourado, L. P. A., et al. (2013). Food components and the immune system: from tonic agents to allergens. Front. Immunol. 4:102. doi: 10.3389/fimmu.2013.00102

Forde, T., De Buck, J., Elkin, B., Kutz, S., van der Meer, F., and Orsel, K. (2013). Contrasting results of culture-dependent and molecular analyses of Mycobacterium avium subsp. paratuberculosis from wood bison. Appl. Environ. Microbiol. 79, 4448-4454. doi: 10.1128/AEM.00995-13

Giongo, A., Gano, K. A., Crabb, D. B., Mukherjee, N., Novelo, L. L., Casella, G., et al. (2011). Toward defining the autoimmune microbiome for type 1 diabetes. ISME J. 5, 82-91. doi: 10.1038/ismej.2010.92

Gophna, U., Sommerfeld, K., Gophna, S., Doolittle, W. F., and van Zanten, S. J. O. (2006). Differences between tissue-associated intestinal microfloras of patients with Crohn's disease and ulcerative colitis. J. Clin. Microbiol. 44, 4136-4141. doi: 10.1128/JCM.01004-06

Graf, D., Di Cagno, R., Fåk, F., Flint, H. J., Nyman, M., Saarela, M., et al. (2015). Contribution of diet to the composition of the human gut microbiota. Microb. Ecol. Health Dis. 26:26164. doi: 10.3402/mehd.v26.26164

Juste, R. A., Elguezabal, N., Garrido, J. M., Pavon, A., Geijo, M. V., Sevilla, I., et al. (2008). On the prevalence of $M$. avium subspecies paratuberculosis DNA in the blood of healthy individuals and patients with inflammatory bowel disease. PLoS ONE 3:e2537. doi: 10.1371/journal.pone.0002537

Karlsson, F., Tremaroli, V., Nielsen, J., and Bäckhed, F. (2013). Assessing the human gut microbiota in metabolic diseases. Diabetes Metab. Res. Rev. 62, 3341-3349. doi: 10.2337/db13-0844

Karunasena, E., McMahon, K. W., Chang, D., and Brashears, M. M. (2014). Host responses to the pathogen Mycobacterium avium subsp. paratuberculosis and beneficial microbes exhibit host sex specificity. Appl. Environ. Microbiol. 80, 4481-4490. doi: 10.1128/AEM.01229-14

Kau, A. L., Ahern, P. P., Griffin, N. W., Goodman, A. L., and Gordon, J. I. (2011). Human nutrition, the gut microbiome and the immune system. Nature 474, 327-336. doi: 10.1038/nature10213

Kennedy, D. J., and Benedictus, G. (2001). Control of Mycobacterium avium subsp. paratuberculosis infection in agricultural species. Rev. Sci. Technol. 20, 151-179.

Knights, D., Lassen, K. G., and Xavier, R. J. (2013). Advances in inflammatory bowel disease pathogenesis: linking host genetics and the microbiome. Gut 62, 1505-1510. doi: 10.1136/gutjnl-2012-303954

Knösel, T., Schewe, C., Petersen, N., Dietel, M., and Petersen, I. (2009). Prevalence of infectious pathogens in Crohn's disease. Pathol. Res. Pract. 205, 223-230. doi: 10.1016/j.prp.2008.04.018

Kuo, S.-M. (2013). The interplay between fiber and the intestinal microbiome in the inflammatory response. Adv. Nutr. 4, 16-28. doi: 10.3945/an.112.003046

Lee, S. C., Tang, M. S., Lim, Y. A. L., Choy, S. H., Kurtz, Z. D., Cox, L. M., et al. (2014). Helminth colonization is associated with increased diversity of the gut microbiota. PLoS Negl. Trop. Dis. 8:e2880. doi: 10.1371/journal.pntd.00 02880 
Ley, R. E., Hamady, M., Lozupone, C., Turnbaugh, P. J., Ramey, R. R., Bircher, J. S., et al. (2008). Evolution of mammals and their gut microbes. Science 320, 1647-1651. doi: 10.1126/science. 1155725

Ling, Z., Kong, J., Liu, F., Zhu, H., Chen, X., Wang, Y., et al. (2010). Molecular analysis of the diversity of vaginal microbiota associated with bacterial vaginosis. BMC Genomics 11:488. doi: 10.1186/1471-2164-11-488

Lozupone, C., and Knight, R. (2005). UniFrac: a new phylogenetic method for comparing microbial communities. Appl. Environ. Microbiol. 71, 8228-8235. doi: 10.1128/AEM.71.12.8228-8235

Magoč, T., and Salzberg, S. (2011). FLASH: fast length adjustment of short reads to improve genome assemblies. Bioinformatics 27, 2957-2963. doi: 10.1093/bioinformatics/btr507

Maio, E., Carta, T., Balseiro, A., Sevilla, I. A., Romano, A., Ortiz, J. A., et al. (2011). Paratuberculosis in european wild rabbits from the iberian peninsula. Res. Vet. Sci. 91, 212-218. doi: 10.1016/j.rvsc.2010.12.014

Manichanh, C., Rigottier-Gois, L., Bonnaud, E., Gloux, K., Pelletier, E., Frangeul, L., et al. (2006). Reduced diversity of faecal microbiota in Crohn's disease revealed by a metagenomic approach. Gut 55, 205-211. doi: 10.1136/gut.2005.073817

Masala, S., Paccagnini, D., Cossu, D., Brezar, V., Pacifico, A., Ahmed, N., et al. (2011). Antibodies recognizing Mycobacterium avium paratuberculosis epitopes cross-react with the beta-cell antigen ZnT8 in Sardinian type 1 diabetic patients. PLOS ONE 6:e26931. doi: 10.1371/journal.pone.00 26931

Morgan, X., Tickle, T., and Sokol, H. (2012). Dysfunction of the intestinal microbiome in inflammatory bowel disease and treatment. Genome Biol. 13:R79. doi: 10.1186/gb-2012-13-9-r7

Mukhopadhya, I., Hansen, R., El-Omar, E. M., and Hold, G. L. (2012). IBD-what role do Proteobacteria play? Nat. Rev. Gastroenterol. Hepatol. 9, 219-230. doi: 10.1038/nrgastro.2012.14

Munyaka, P. M., Sepehri, S., Ghia, J. E., and Khafipour, E. (2016). Carrageenan gum and adherent invasive Escherichia coli in a piglet model of inflammatory bowel disease: impact on intestinal mucosa-associated microbiota. Front. Microbiol. 7:462. doi: 10.3389/fmicb.2016.00462

Naser, S., Thanigachalam, S., Dow, C., and Collins, M. (2013). Exploring the role of Mycobacterium avium subspecies paratuberculosis in the pathogenesis of type 1 diabetes mellitus: a pilot study. Gut Pathog. 5:14. doi: 10.1186/1757-4749-5-14

Naser, S. A., Ghobrial, G., Romero, C., and Valentine, J. F. (2004). Culture of Mycobacterium avium subspecies paratuberculosis from the blood of patients with Crohn 's disease. Lancet 364, 1039-1044. doi: 10.1016/S01406736(04)17058-X

Nicodemus, N., Pérez-Alba, L., Carabaño, R., Blas, C. de Badiola, I., Pérez de Rozas, A., et al. (2005). "Effect of level of fibre and level of ground of fibre sources on digestion and ileal and caecal characterization of microbiota of early weaned rabbits," in Proceedings of the 8th World Rabbit Congress, (Pueblo: World Rabbit Science Association), 928-929. Available at:http://www.cabdirect.org/abstracts/20053160665.html;jsessionid=478A40C F716A 3BF9247C18EC287C6755 [accessed on September 7-10, 2004].

Ooi, J. H., Waddell, A., Lin, Y.-D., Albert, I., Rust, L. T., Holden, V., et al. (2014). Dominant effects of the diet on the microbiome and the local and systemic immune response in mice. PLOS ONE 9:e86366. doi: 10.1371/journal.pone.0086366

Price, M., Dehal, P., and Arkin, A. (2010). FastTree 2-approximately maximum-likelihood trees for large alignments. PLOS ONE 5:e9490. doi: 10.1371/journal.pone.0009490

Quast, C., Pruesse, E., Yilmaz, P., Gerken, J., Schweer, T., Yarza, P., et al. (2013). The SILVA ribosomal RNA gene database project: improved data processing and web-based tools. Nucleic Acids Res. 41, D590-D596. doi: 10.1093/nar/gks1219

Rees Davies, R., Rees Davies, J. A. E., Davies, R. R., and Rees Davies, J. A. E. (2003). Rabbit gastrointestinal physiology. Vet. Clin. North Am. Exot. Anim. Pract. 6, 139-153. doi: 10.1016/S1094-9194(02)00024-5

Rienzi, S., Sharon, I., Wrighton, K., and Koren, O. (2013). The human gut and groundwater harbor non-photosynthetic bacteria belonging to a new candidate phylum sibling to Cyanobacteria. Elife 2:e01102. doi: 10.7554/eLife. 01102

Sato, T., Matsumoto, K., Okumura, T., Yokoi, W., Naito, E., Yoshida, Y., et al. (2008). Isolation of lactate-utilizing butyrate-producing bacteria from human feces and in vivo administration of Anaerostipes caccae strain L2 and galactooligosaccharides in a rat model. FEMS Microbiol. Ecol. 66, 528-536. doi: 10.1111/j.1574-6941.2008.00528.x
Schuster, S. C. (2008). Next-generation sequencing transforms today's biology. Nat. Methods 5, 16-18. doi: 10.1038/nmeth1156

Segain, J., Blétiere, D. D., and Bourreille, A. (2000). Butyrate inhibits inflammatory responses through NFKB inhibition: implications for Crohn's disease. Gut 47, 397-403. doi: 10.1136/gut.47.3.397

Serino, M., Luche, E., Gres, S., Baylac, A., Berge, M., Cenac, C., et al. (2011). Metabolic adaptation to a high-fat diet is associated with a change in the gut microbiota. Gut 61, 543-553. doi: 10.1136/gutjnl-2011-301012

Sevilla, I. A., Garrido, J. M., Molina, E., Geijo, M. V., Elguezabal, N., Vázquez, P., et al. (2014). Development and evaluation of a novel multicopyelement-targeting triplex PCR for detection of Mycobacterium avium subsp. paratuberculosis in feces. Appl. Environ. Microbiol. 80, 3757-3768. doi: 10.1128/AEM.01026-14

Shanmugam, M., Sethupathi, P., Rhee, K.-J., Yong, S., and Knight, K. L. (2005). Bacterial-induced inflammation in germ-free rabbit appendix. Inflamm. Bowel. Dis. 11, 992-996. doi: 10.1097/01.MIB.0000182869.74648.0f

Soo, R. M., Skennerton, C. T., Sekiguchi, Y., Imelfort, M., Paech, S. J., Dennis, P. G., et al. (2014). An expanded genomic representation of the phylum cyanobacteria. Genome Biol. Evol. 6, 1031-1045. doi: 10.1093/gbe/evu073

Stecher, B., and Hardt, W.-D. (2011). Mechanisms controlling pathogen colonization of the gut. Curr. Opin. Microbiol. 14, 82-91. doi: 10.1016/j.mib.2010.10.003

Stein, R. R., Bucci, V., Toussaint, N. C., Buffie, C. G., Rätsch, G., Pamer, E. G., et al. (2013). Ecological modeling from time-series inference: insight into dynamics and stability of intestinal microbiota. PLoS Comput. Biol. 9:e1003388. doi: 10.1371/journal.pcbi.1003388

Steinle, N., Cirimotch, S., Ryan, K., Fraser, C., Shuldiner, A., and Mongodin, E. (2013). Increased gut microbiome diversity following a high fiber mediterranean style diet. FASEB J. 27:1056.

Terrapon, N., and Henrissat, B. (2014). How do gut microbes break down dietary fiber? Trends Biochem. Sci. 39, 156-158. doi: 10.1016/j.tibs.2014.02.005

Trygg, J., and Wold, S. (2002). Orthogonal projections to latent structures (O-PLS). J. Chemom. 16, 119-128. doi: 10.1002/cem.695

Vaughan, J. A., Lenghaus, C., Stewart, D. J., Tizard, M. L., and Michalski, W. P. (2005). Development of a Johne's disease infection model in laboratory rabbits following oral administration of Mycobacterium avium subspecies paratuberculosis. Vet. Microbiol. 105, 207-213. doi: 10.1016/j.vetmic.2004.10.019

Walker, A. W., Sanderson, J. D., Churcher, C., Parkes, G. C., Hudspith, B. N., Rayment, N., et al. (2011). High-throughput clone library analysis of the mucosa-associated microbiota reveals dysbiosis and differences between inflamed and non-inflamed regions of the intestine in inflammatory bowel disease. BMC Microbiol. 11:7. doi: 10.1186/1471-2180-11-7

Warwick, R., and Clarke, K. (2006). PRIMER 6. Prim. Ltd Plymouth. Available at: http://www.primer-e.com

Wen, L., Ley, R. E., Volchkov, P. Y., Stranges, P. B., Avanesyan, L., Stonebraker, A. C., et al. (2008). Innate immunity and intestinal microbiota in the development of Type 1 diabetes. Nature 455, 1109-1113. doi: 10.1038 /nature07336

$\mathrm{Xu}, \mathrm{Z}$., and Knight, R. (2014). Dietary effects on human gut microbiome diversity. Br. J. Nutr. 113, S1-S5. doi: 10.1017/S0007114514004127

Zeng, B., Han, S., Wang, P., Wen, B., Jian, W., Guo, W., et al. (2015). The bacterial communities associated with fecal types and body weight of rex rabbits. Sci. Rep. 5:9342. doi: 10.1038/srep09342

Zhu, Y., Wang, C., and Li, F. (2015). Impact of dietary fiber/starch ratio in shaping caecal microbiota in rabbits. Can. J. Microbiol. 61, 771-784. doi: 10.1139/cjm2015-0201

Conflict of Interest Statement: The authors declare that the research was conducted in the absence of any commercial or financial relationships that could be construed as a potential conflict of interest.

Copyright (c) 2016 Arrazuria, Elguezabal, Juste, Derakhshani and Khafipour. This is an open-access article distributed under the terms of the Creative Commons Attribution License (CC BY). The use, distribution or reproduction in other forums is permitted, provided the original author(s) or licensor are credited and that the original publication in this journal is cited, in accordance with accepted academic practice. No use, distribution or reproduction is permitted which does not comply with these terms. 